\title{
Circular Colouring for Emission Minimalization
}

\author{
R. Zewdie ${ }^{*}$ J. Hora \\ Faculty of Engineering, Czech University of Life Sciences, Prague, Czech Republic \\ *Corresponding author: Zewdie@tf.czu.cz \\ Y. Nigussie \\ Department of Mathematics, East Tennessee State University, Johnson City, USA
}

DOI: 10.2478/v10158-012-0043-y

\begin{abstract}
The standard time allocation of classic road junctions in major towns and cities are often the main indicators of the government policy and management strategy in reducing traffic jams with its associated emission production from the traffic flow. In this paper, we apply the circular colouring method of Graph Theory (Vince, 1988; Zhu, 2001), to the emission reduction problem at a traffic road junction. The result of this paper is addressing this problem by optimizing the waiting time interval for two selected models. A traffic road junction is modelled as a finite graph $G=(V, E)$. Each vertex $v$ of $G$ represents a given traffic light. Two vertices $\mathrm{v}$ and $\mathrm{u}$ are connected with an edge if their corresponding represented traffic flows collide and each time in such case they are given an overlapping green light time interval.
\end{abstract}

KEY WORDS: Circular colouring, traffic flow, circular chromatic number.

\section{INTRODUCTION}

In day to day life, in every major town or city the problem of human - machine interaction has been a major issue for decades. Vehicles, automobiles and other self-propelled machines play a vital role in the economic infrastructure of both state and private companies. However, the communication network and road capacities of major cities are not efficiently solved. In most cases, this leads to catastrophic traffic jams on road junctions due to ineffective traffic light arrangements. This paper is expected to give guidelines and emphasis on how to minimize emissions at different types of classic road traffic junctions applying the circular colouring method (Zhu, 2001). This paper shows a partial improvement in network and traffic management policies which might contribute to reducing carbon emissions and associated problems such as public transport priority, traffic signal control and coordination, as well as their layout. The development of Intelligent Transport systems and associated improvements in urban traffic control quality has a crucial role in the road traffic junction quality impact of road traffic.

Polluting emissions, aggravated by traffic composition, i.e. the number of vehicles and their categories, the drivers' experience as well as their behavior, seasonal weather, gradients and the technical conditions of vehicles and the waiting time at traffic lights, are the burning issues for the individual management and government policies of a given country or institution. For effective traffic management strategies, we have to investigate 
the relationship between traffic flow, the emission pollutants and dispersion in the atmosphere and the potential impact on the processes of the network and traffic management policies.

In this paper, we assume a graph $\mathrm{G}$ is finite and simple; (no loops and multiple edges). More specifically, a graph $G=(V, E)$ is a pair, such that $V$ is a finite set of vertices and $E$ is the set of edges such that $\mathrm{E} \subseteq \mathrm{V} \times \mathrm{V}$ (unordered pair). If $\mathrm{u}$ and $\mathrm{v}$ are connected by an edge, then we write $\mathrm{uv} \in \mathrm{E}$.

Let $n$ be a positive integer. Consider a crossroad with $n$ traffic lights. Let $v_{1}, v_{2}, v_{n}$ denote the $n$ traffic lights.

Definition: We say $v_{i}$ and $v_{j}$ are incompatible if assigning an overlapping green light time to $\mathrm{v}_{\mathrm{i}}$ and $\mathrm{v}_{\mathrm{j}}$ green causes a traffic collision.

Then, given a crossroad $A$, an undirected graph $G=(V, E)$ corresponding to $A$ is constructed as follows: The set of vertices is the set of traffic lights $V=\left\{v_{1}, v_{2}, v_{n}\right\}$ and the set of edges is the set of incompatible pairs of vertices. That is, $E=\left\{v_{i} v_{j}: v_{i}\right.$ and $v_{j}$ are incompatible\}.

Therefore, cars are allowed to proceed by $\mathrm{v}_{\mathrm{i}}$ and $\mathrm{v}_{\mathrm{j}}$, while $\mathrm{v}_{\mathrm{i}} \mathrm{v}_{\mathrm{j}} \in \mathrm{E}$ avoid collision if $\mathrm{v}_{\mathrm{i}}$ and $\mathrm{v}_{\mathrm{j}}$ are allocated to non-overlapping time intervals. This is where circular colouring plays a crucial role, as we shall see shortly.

\section{OBJECTIVE}

This paper examines the character of two models of classic road junctions. Model I is presented as a twelve traffic light road junction and model II as an eleven traffic light road junction. These two models appear in almost all major towns and cities. For the traffic optimization of our selected two models, the authors applied Graph Theory, in particular the "circular colouring" method. This corresponds to what is known as the "circular chromatic number", $\chi_{c}(\mathrm{G})$ of a given graph $\mathrm{G}$, which we will formally define in Section 4.

\section{MODEL I: CLASSIC FOUR WAY TWELVE TRAFFIC LIGHT ROAD JUNCTION}

There are four roads in Figure 1, with 4 orientations, for example north, south, east and west. Each road has three directions: left turn, straight and right turn. Lights number 1, 2 and 3 correspond to left, straight and right turn, respectively. A similar pattern applies to lights numbered 4, 5, 6; 7, 8, 9 and 10, 11, 12 (see Figure 1). Figure 1 also demonstrates how traffic light number 1 is in a collision course with 4 traffic lights, namely traffic lights $4,8,10$ and 11. 


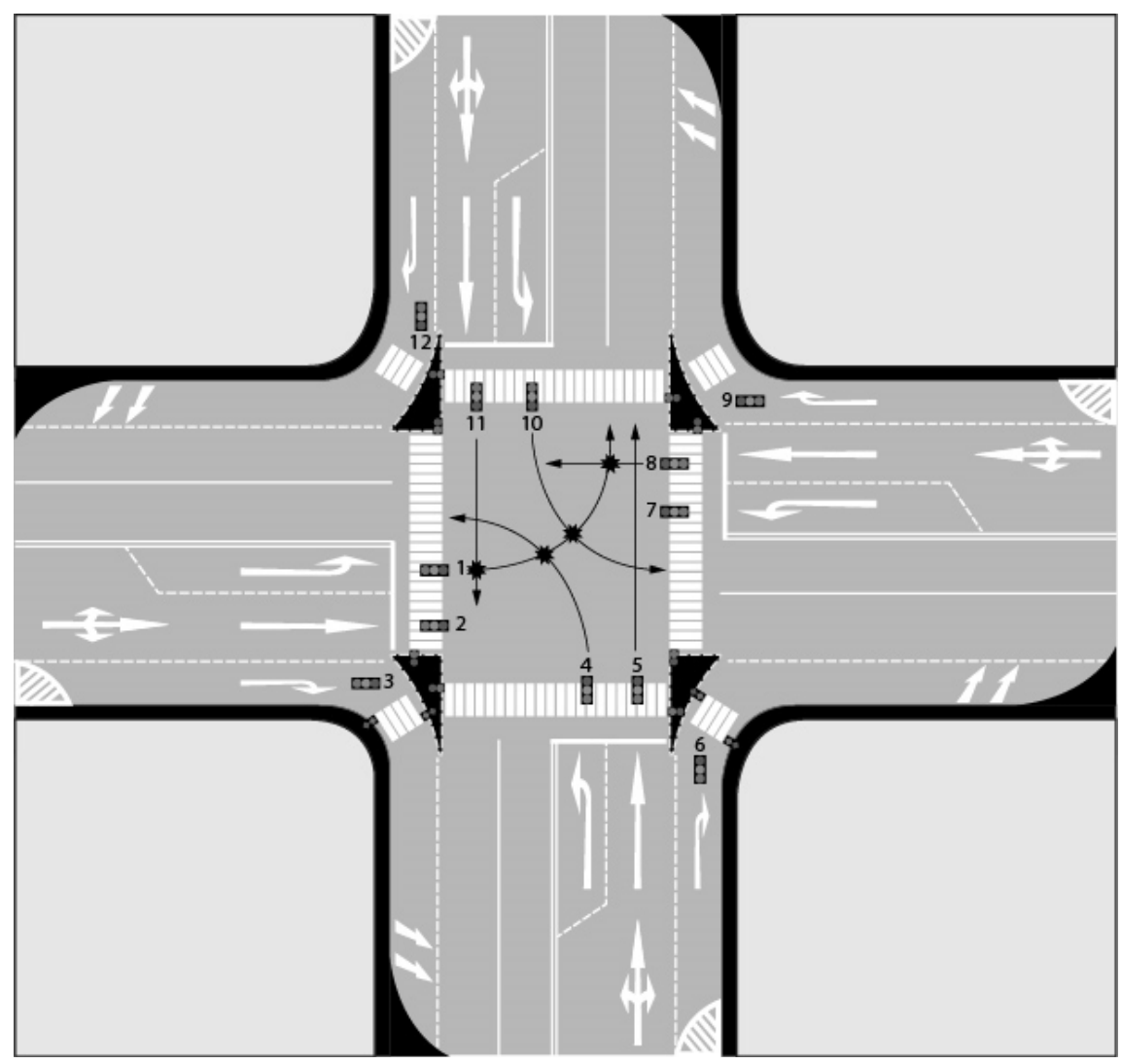

Figure 1: Model I: a classic road junction with twelve vertices.

The following graph (Figure 2) is derived from the above four way classic road junction (Figure 1). This graph has twelve vertices. Each vertex represents a specific traffic light. Recall that the adjacency of two vertices $u$ and $v$ implies $u$ and $v$ are incompatible.

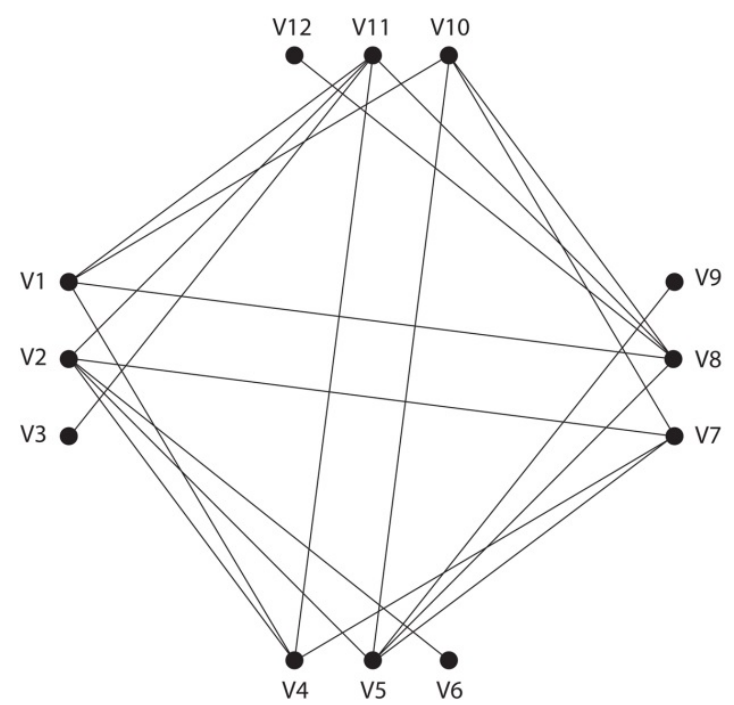

Figure 2: A Graph for model I, with 12 vertices (lights) and edges connecting incompatible vertices.

Vertex 1 (left turn) is adjacent to vertices 4, 8, 10 and 11; (as depicted in Figure 1 a vehicle moving from position number 1 is incompatible with vehicles moving from positions 4, 8, 10 
and 11); vertex 2 (straight) is adjacent to vertices 4, 5, 6, 7 and 11; vertex 3 (right turn) is adjacent only to vertex number 11 . This goes on in the same pattern (by symmetry) as it applies for the whole cycle up to vertex number 12, as shown in Figure 2.

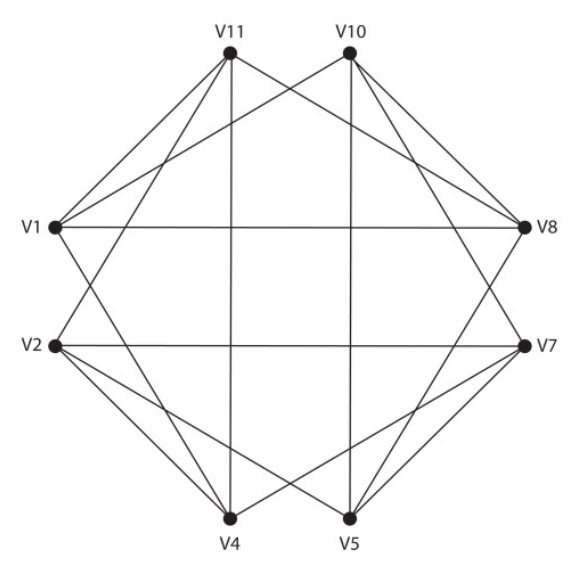

Figure 3: Reduced graph $G_{1}$ of model $I$ with 8 adjacent vertices.

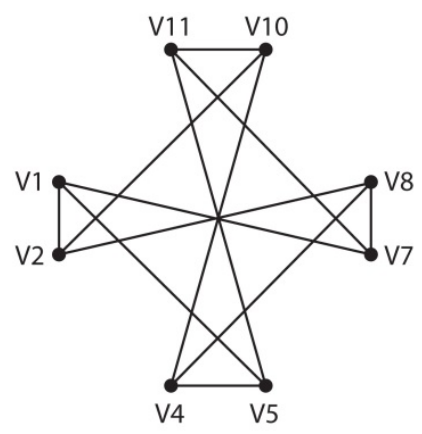

Figure 4: Model I: The complement graph $\mathrm{G}_{1}$ ' of $\mathrm{G}_{1}$; (to Figure 3 graph of incompatibility).

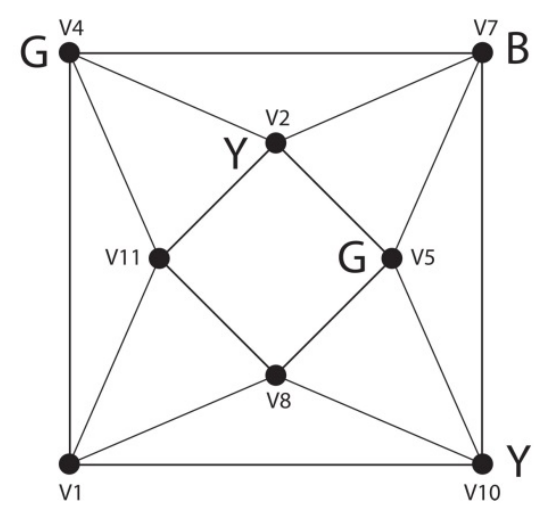

Figure 5: Model I: A symmetric representation of $\mathbf{G}_{1}$.

\section{CIRCULAR CHROMATIC NUMBER OF A GRAPH}

DEFINITION: The "chromatic number", $\chi(\mathrm{G})$ of a graph $\mathrm{G}$ is the minimum number of colours required to colour $G$ properly. By proper colouring, we mean no two adjacent (connected by an edge) vertices should get the same colour. 
In a traffic flow, proper colouring corresponds to requiring no two incompatible traffic lights should have an overlapping green light time interval. The number of colours used to colour a graph $\mathrm{G}$ corresponds to the total of one complete traffic period. That is, a period where each light gets exactly one green light interval. In other words, the maximum length a car has to wait before it gets a green light to proceed. Therefore the "chromatic number" seems to be an optimal solution since it minimizes the number of colours. In other words $\chi(\mathrm{G})$ is a complete traffic period length. For example, the "chromatic number" if each green light time is a half minute interval and if we colour $\mathrm{G}$ with 4 colours, then the total traffic period is 2 minutes. However, we shall show in Section 5 that this is not the case.

Denote by $S_{p}$ the circle in $R^{2}$ (the usual $X-Y$ plane) with circumference $p$ centered at the origin of $\mathrm{R}^{2}$ and by $A\left(S_{p}\right)$ the set of open arcs of $S_{p}$.

DEFINITION: Let $\mathrm{G}=(\mathrm{V}, \mathrm{E})$ be an undirected graph without loops. A circular colouring of $G$ is a mapping $c_{p}: V \rightarrow A\left(S_{p}\right)$, such that the length of $c(v)$ is (greater or) equal to 1 (and in general the weight of the vertex $v$ ) and $c_{p}(v) \cap c_{p}(w)$ is empty whenever $v$ and $w$ are adjacent in $\mathrm{G}(\mathrm{vw} \in \mathrm{E}(\mathrm{G}))$.

DEFINITION: Circular chromatic number $\chi_{c}(G)$ of a graph $G=(V, E)$ is the infimum of all real numbers $p$, for which a circular colouring $c_{p}$ of $G$ exists.

There are several cases where $\chi_{c}(\mathrm{G})$ is strictly less than $\chi(\mathrm{G})$. Therefore, $\chi_{c}(\mathrm{G})$ is more efficient than $\chi(\mathrm{G})$ as is shown in this paper. In other words, the total one traffic cycle period can be less if we use $\chi_{c}(\mathrm{G})$ instead of $\chi(\mathrm{G})$.

The classic approach to set a stop-go cycle for a crossroad is to determine the chromatic number $\chi(\mathrm{G})$ of the graph and to assign an interval to every colour in the minimal colouring of G. Since this approach is not the best with respect to the time needed for an entire cycle, the circular chromatic number of a graph is used. In general, every vertex in $G$ has a weight which corresponds to the time for which the corresponding direction is open. This is necessary if the number of cars coming from different directions is not even. In this paper, for simplicity, we give the definition assuming that all the weights are equal to 1 .

It is easy to see (Zhu, 2001) that the "circular chromatic number" of a graph is less or equal to its chromatic number:

Proposition 1. $\chi_{c}(\mathrm{G}) \leq \chi(\mathrm{G})$.

It is shown in Zhu (2001) that this infimum is attained and thus we can replace it with the minimum. In what follows an $\operatorname{arc} c_{p}(v)$ will be usually identified (and drawn in Figures) with its centre point. Thus, in the circular colouring of $G$ the distance (along the circle) of two centre points corresponding to vertices connected by an edge must be at least 1 (given the arcs are non overlapping).

Now consider the crossroad in Figure 1. Since there are two lanes in each direction, we shall assume that cars coming from direction 1 and 5 can go simultaneously. By symmetry, the same is true for directions 4 and 9, 7 and 11, 10 and 2. The corresponding graph is in Figure 2.

A vertex $\mathrm{v}$ is called "pendant" if it is adjacent to only one vertex (degree 1). Vertices number 3, 6, 9 and 12 are pendants. A pendant vertex $v$ in $G$ can be identified with a vertex of $G$ that has a common neighbor with $v$, without changing $\chi_{c}(G)$ and $\chi(G)$. Therefore, we can omit pendants when we study the (circular) chromatic number. As a result, we get the "reduced graph" $\mathrm{G}_{1}$ depicted in Figure 3.

Proposition 2. The chromatic number of the graph $\mathrm{G}_{1}$ is $4\left(\chi\left(\mathrm{G}_{1}\right)=4\right)$.

Proof: First we prove that $\chi\left(\mathrm{G}_{1}\right) \leq 4$, by 4-colouring $\mathrm{G}_{1}$ with a colouring function c. (see Figure 2 ).

Let $c\left(v_{1}\right)=c\left(v_{2}\right)=c\left(v_{3}\right)=R \quad$ (R for Red)

$c\left(v_{4}\right)=c\left(v_{5}\right)=c\left(v_{6}\right)=B \quad$ (B for Blue) 


$$
\begin{array}{ll}
c\left(v_{7}\right)=c\left(v_{8}\right)=c\left(v_{9}\right)=G \quad(G \text { for Green }) \\
c\left(v_{10}\right)=c\left(v_{11}\right)=c\left(v_{12}\right)=Y & (Y \text { for Yellow })
\end{array}
$$

Therefore: $\chi\left(\mathrm{G}_{1}\right) \leq 4$.

Now, we prove that the graph has no 3-colouring (see the symmetric representation of $\mathrm{G}_{1}$ in Figure 5). Suppose, without loss of generality, that there is a 3-colouring $c^{*}$ using Blue (B), Green $(G)$ and Yellow $(Y)$. We may assume $c^{*}\left(v_{7}\right)=B$ and $c^{*}\left(v_{4}\right)=G$. Now, this forces $c^{*}\left(v_{2}\right)=Y$ (since $c^{*}\left(v_{2}\right)$ is adjacent to both $v_{7}$ and $\left.v_{4}\right)$. Similarly, this forces that $c^{*}\left(v_{5}\right)=G$. Then $\mathrm{C}^{*}\left(\mathrm{v}_{10}\right)=\mathrm{Y}$. But then $\mathrm{v}_{8}$ and $\mathrm{v}_{11}$ both have to be $\mathrm{B}$, which is a contradiction, since $\mathrm{v}_{8}$ and $\mathrm{v}_{11}$ are adjacent. Therefore, there is no such 3-colouring $\mathrm{c}^{*}$ of $\mathrm{G}_{1}$.

Therefore: $\chi\left(\mathrm{G}_{1}\right) \geq 4$. We deduce that $\chi\left(\mathrm{G}_{1}\right)=4$.

\section{Proposition 3. The circular chromatic number of the graph $G_{1}$ is $4 ; \chi_{c}\left(G_{1}\right)=4$}

Proof: By Propositions 1 and 2 we have that the circular chromatic number is at most 4.

Now, we prove that it is equal to 4 . In any circular colouring the $\operatorname{arcs} c_{p}(v)$ and $c_{p}(w)$ can intersect only if $v$ and $w$ are not adjacent in $G_{1}$. Consider the graph $G_{1}$ ' (Figure 4), the complement of the graph $G_{1}$, i.e., vertices $v$ and $w$ are adjacent in $G_{1}$ ' if and only if they are not adjacent in $G_{1}$. Since there is no triangle (three vertices pairwise connected) in $G_{1}$ ' there are no three vertices in $G_{1}$ such that their corresponding arcs have a non-empty intersection. Thus, every point on the circle is contained in at most two arcs. Since there are eight arcs of length one, the circumference of the circle must be at least 4 .

This is an example where the circular chromatic number is identical to the chromatic number. However, in the next section, we show a model for which the circular chromatic number is clearly a more efficient method.

\section{MODEL II: CLASSIC FOUR WAY ELEVEN LIGHT ROAD JUNCTION}

Now, we shall examine the situation with one less direction. Consider the same crossroad as in Figure 1, but omit the possibility of turning left $\left(\mathrm{v}_{1}\right)$ as depicted in Figure 6. We get the same graph $G_{2}$ which is obtained from $G_{1}$ by deleting $v_{1}$ from $G_{1}$. Again, we can study the reduced graph by omitting the vertices of degree 1 . We get the graph $G_{2}$ with seven vertices (Figure 7). By symmetry of $\mathrm{G}_{1}$ the statements about $\mathrm{G}_{2}$ would be true if we would omit an arbitrary left or straight direction from the original 12-direction crossroad.

Proposition 4. The chromatic number of the graph $G_{2}$ is $4 ; \chi_{c}\left(G_{2}\right)=4$

Proof: The proof is in all respects the same as the proof of Proposition 2, since the proof of $\chi(\mathrm{G} 1)>3$ is independent of the vertex $\mathrm{v}_{1}$.

Proposition 5. The circular chromatic number of the graph $\mathrm{G}_{2}$ is 7/2; $\chi_{c}\left(G_{2}\right)=7 / 2$.

Prooof: The 7/2 circular colouring shown in Figure 10 proves that the circular chromatic number is at most 7/2. By the same argument as in the proof of Proposition 3, no three arcs can overlap, and since there are seven arcs, the circular chromatic number is at least $7 / 2$. Hence, $\chi_{c}\left(G_{2}\right)=7 / 2$. 


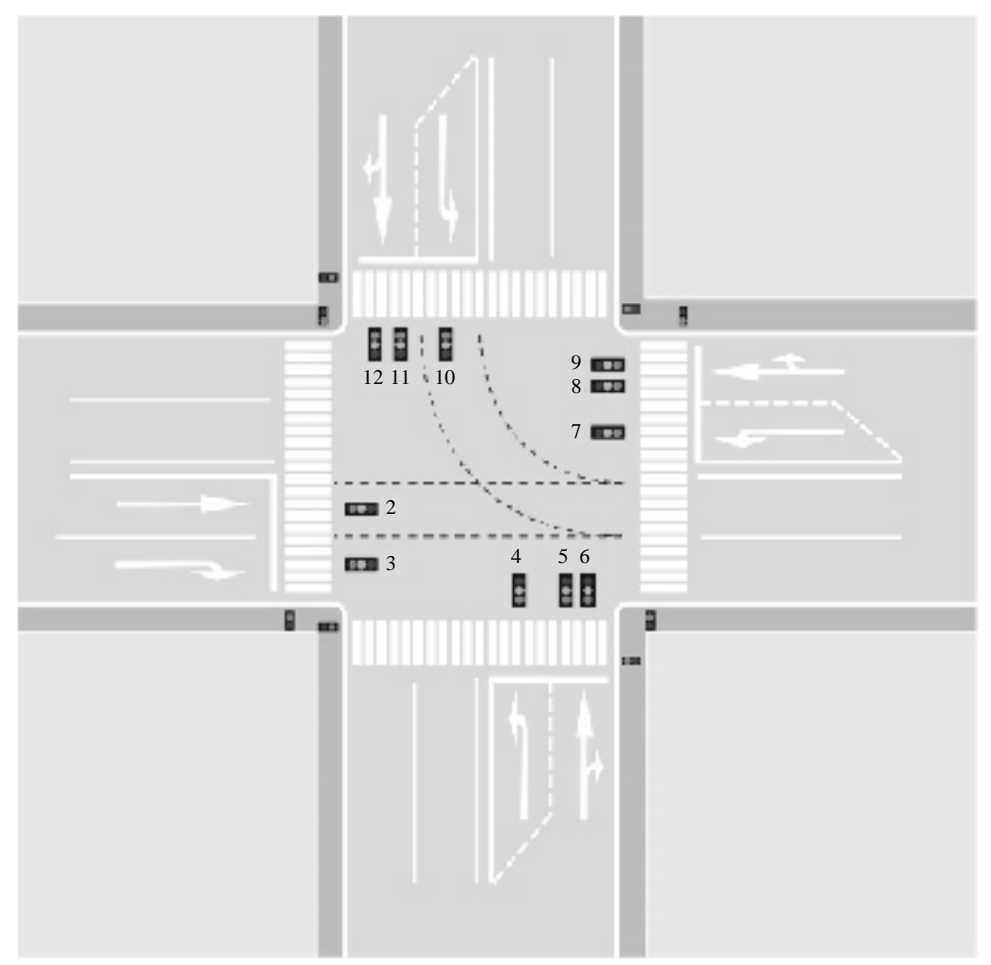

Figure 6: Model II: A classic road junction with eleven vertices.

To see the validity of the $7 / 2$ colouring of $G_{2}$ given in Figure 10, it suffices to verify (using Figure 11) that the following two constraints are satisfied:

- Every traffic light, $\left(\mathrm{v}_{2}, \mathrm{v}_{3}, \ldots\right.$, and $\left.\mathrm{v}_{10}\right)$ has received at least one full green light period;

- No overlapping green light time is assigned to any incompatible pair.

The fact that the total one traffic period is $7 / 2$ time units is rather straight forward from either of the two figures.

Note also that the traffic lights of Model II that are reduced, $v_{3}, v_{6}, v_{9}$ and $v_{12}$, (see Figure 7); any amount of green light interval that does not overlap with $\mathrm{v}_{11}, \mathrm{v}_{2}, \mathrm{v}_{5}$ and $\mathrm{v}_{8}$, respectively can now be assigned.

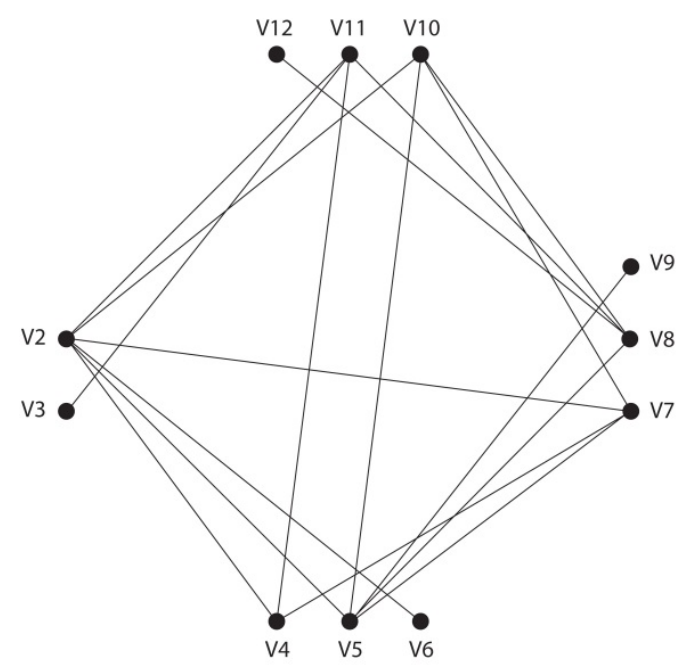

Figure 7: A graph for model II with 11 vertices (11 lights) and edges connecting incompatible vertices. 


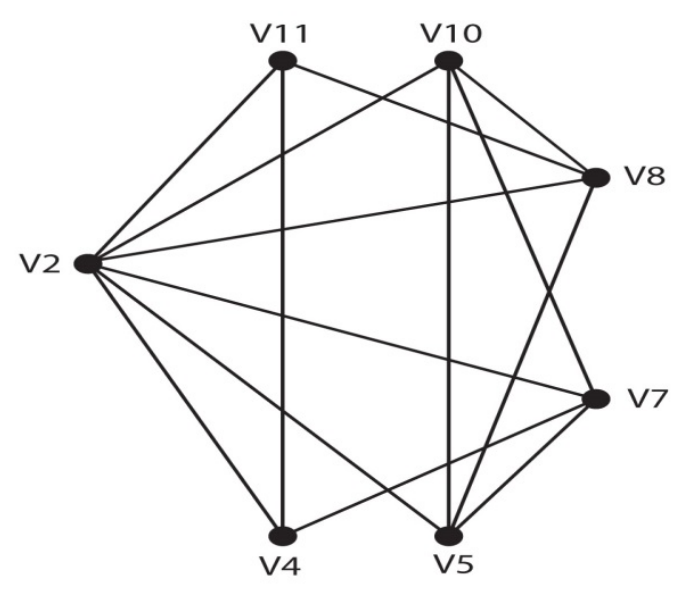

Figure 8: $\mathbf{G r a p h} \mathbf{G}_{2}$.

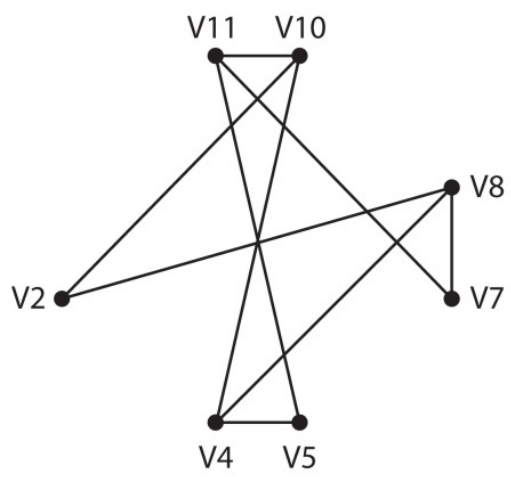

Figure 9: Complement $G_{2}$ ' of $G_{2}$.

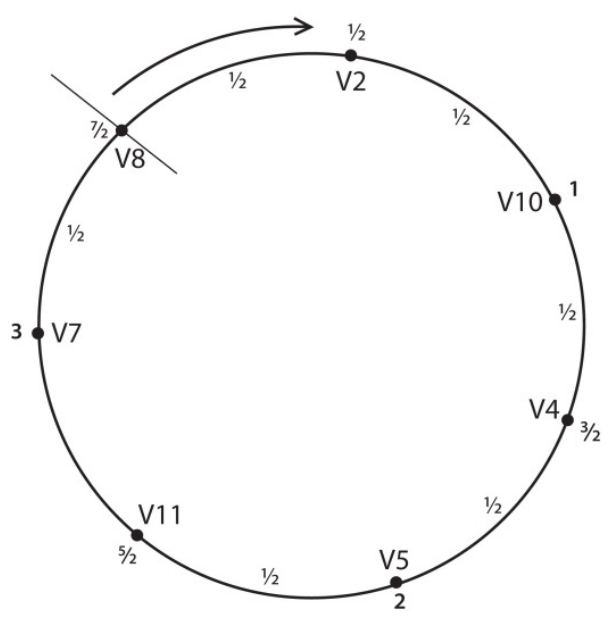

Figure 10: Circular colouring of $G_{2}$. 


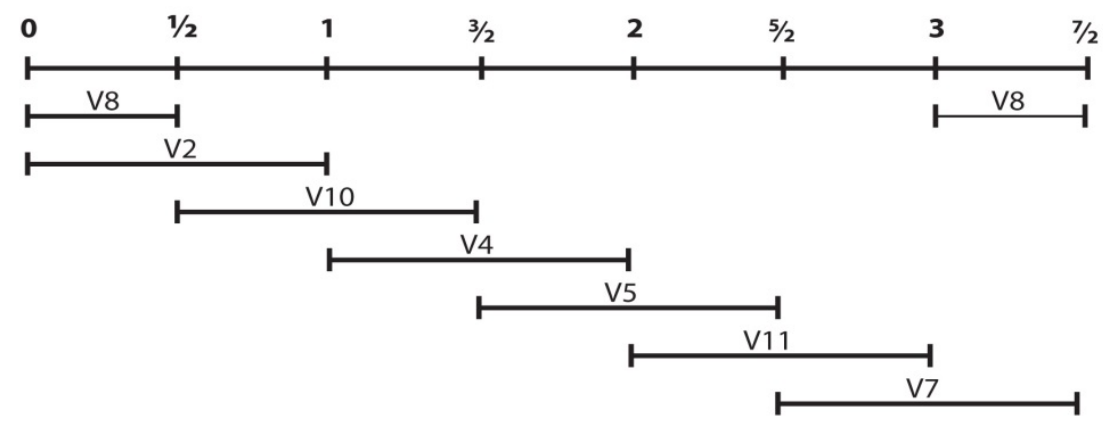

Figure 11: Traffic flow phases after chromatic circular graph.

\section{CONCLUSION}

In this paper we have chosen the two most common traffic junctions and showed in one of the two cases that the circular colouring method yields a more efficient solution than the usual colour minimization method. To be more specific, we have first reduced the traffic model by removing a redundant traffic light. Interestingly, such a traffic light reduction alone does not result in any traffic period reduction as shown by Proposition 4 . On the other hand Proposition 2 also shows that the circular colouring method alone does not necessarily yield a better solution. Thus, it is made apparent in this paper that it is the combination of both methods that translates into more efficient traffic management.

By virtue of simplicity, we have made our point clear without including long and tedious calculations. From a real world point of view, however, it is often necessary to consider additional factors. These factors inevitably lead to a more technical and computational analysis. We discuss here two such important factors and we conclude by remarking how we intend to resolve them.

In the first place, all roads at a traffic junction need not have an equal distribution of traffic load. A given road may be a lot busier than another although they meet at the same junction. As a result assigning equal time length to all traffic lights can lead to some undesirable traffic congestion.

The second factor can be described as follows: We note that we have defined two traffic lights to be either incompatible or compatible. From this point of view, we decided to allow an overlapping green light interval only to compatible lights. However, we did not specify how much of an overlap time that can be allowed. The maximum being one, we have left the minimum overlapping time open to any. On the other hand, when two lights are incompatible, we have only considered the case that they are assigned a disjoint (nonoverlapping time) interval. However, in a real world scenario, the situation may not be that simple. For instance, in order to assure the safety of the traffic light system, there may be an additional requirement that two incompatible lights should not only have a non-overlapping green light time, but also that there is a certain time gap between the intervals. That is, one may not be given a green light immediately after the other's green light turns red, but rather should wait a certain length of time before being allowed to proceed via a green light. There may also exist incompatible pairs of lights where such a restriction is not necessary. This leads to the notion of the "degree of incompatibility" between two incompatible lights. We can also analyze the dual problem of "degree of compatibility" problem between two compatible lights. 
How do we approach such issues? There are certain generalizations of the circular colouring that are suitable to such a variety of real world problems. For the first factor that we discussed above, a type of circular colouring called "vertex-weighted" circular-colouring seems to be appropriate. The busier a given road is, the more weight we give to the light that corresponds to the busy road. In this manner, we obtain a graph which has labels on its vertices. The labels correspond to the weight that traffic light is given. Then, the vertexweighted circular colouring assigns a green light time interval to each traffic light proportional to the given weight of the specific vertex. In short, the vertex-weighted circular colouring addresses the problem of an unequal traffic distribution junction problem.

For the second factor that we discussed above, a type of circular colouring called "edgeweighted" circular-colouring seems to be the appropriate approach. The further apart we want two incompatible lights to have green light intervals, the more weight is given to the edge that is between the corresponding vertices in the graph. In this manner, we obtain a graph which has labels on its edges. The labels correspond to the "degree of incompatibility" between the traffic lights that are represented by the endpoints of the edge. Then, the edgeweighted circular colouring assigns a green light time interval to each traffic light proportional to the given weight of the specific edge.

Each of the edges and vertex-weighted circular-colouring minimizes the colouring number, and so it is presumed to give an optimal solution.

We hope to study and present an extension of the current work in a forthcoming paper, by considering these two types of generalized circular-colourings and exhibit certain models where such methods prove to be more efficient than standard methods.

\section{ACKNOWLEDGEMENT:}

This paper is supported by the Ministry of Education of the Czech Republic, project LD11049 "The transport energy consumption in urban and suburban areas", under a project EU COST TU0902 "integrated assessment technologies to support the sustainable development of urban areas” (2011 - 2013).

\section{REFERENCES}

Abbott, H. L, Zhou, B., 1993. The star chromatic number of a graph. Journal of Graph Theory, 17 (3), pp. 349-360.

Mohar, B., 2001. Circular colouring of edge - weighted Graphs. Department of Mathematics, University of Ljubljana.

Vince, A., 1988. Star chromatic number. Journal of Graph Theory, 12 (4), pp. 551-559.

West, D. B., 1996. Introduction to Graph Theory. Prentice Hall.

Zhu, X., 2001. Circular chromatic number: a survey. Discrete Mathematics, 229, pp. 371-410. 